\title{
A multiparameter model for non-invasive detection of hypoglycemia
}

Ole Elvebakk ${ }^{1}$, Christian Tronstad ${ }^{1}$, Kåre I. Birkeland ${ }^{2,3}$, Trond G. Jenssen ${ }^{2,3,4}$, Marit R. Bjørgaas ${ }^{5,6}$, Hanne L. Gulseth ${ }^{7,8}$, Håvard Kalvøy ${ }^{1}$, Jan O. Høgetveit ${ }^{1,9}$ and $\varnothing$ rjan G. Martinsen ${ }^{9,1}$

${ }^{1}$ Department of Clinical and Biomedical Engineering, Oslo University Hospital, Oslo, Norway

${ }^{2}$ Department of Transplantation Medicine, Oslo University Hospital, Oslo, Norway

${ }^{3}$ Institute of Clinical Medicine, University of Oslo and Oslo University Hospital

${ }^{4}$ Metabolic and Renal Research Group, UiT The Arctic University of Norway, Troms $\varnothing$, Norway

${ }^{5}$ Department of Endocrinology, St. Olavs Hospital, Trondheim University Hospital, Trondheim, Norway.

${ }^{6}$ Department of Clinical and Molecular Medicine, Faculty of Medicine and Health Sciences, NTNU Norwegian University of Science and Technology, Trondheim, Norway.

${ }^{7}$ Department of Chronic Diseases and Ageing, Norwegian Institute of Public Health, Oslo Norway

${ }^{8}$ Department of Endocrinology, Morbid Obesity and Preventive Medicine, Oslo University Hospital, Oslo, Norway

${ }^{9}$ Department of Physics, University of Oslo, Oslo, Norway.

\begin{abstract}
Severe hypoglycemia is the most serious acute complication for people with type 1 diabetes (T1D). Approximately $25 \%$ of people with T1D have impaired ability to recognize impending hypoglycemia, and nocturnal episodes are feared. We have investigated the use of non-invasive sensors for detection of hypoglycemia based on a mathematical model which combines several sensor measurements to identify physiological responses to hypoglycemia. Data from randomized singleblinded euglycemic and hypoglycemic glucose clamps in 20 participants with type 1 diabetes and impaired awareness of hypoglycemia was used in the analyses. Using a sensor combination of sudomotor activity at three skin sites, ECG-derived heart rate and heart rate corrected QT interval, near-infrared and bioimpedance spectroscopy; physiological responses associated with hypoglycemia could be identified with an F1 score accuracy up to $88 \%$. We present a novel model for identification of non-invasively measurable physiological responses related to hypoglycemia, showing potential for detection of moderate hypoglycemia using a wearable sensor system.
\end{abstract}




\section{Introduction}

Even with adequate monitoring and treatment, people with type 1 diabetes (T1D) have higher morbidity and mortality than the general population (Livingstone et al., 2012; Secrest et al., 2010; Lind et al., 2014). In particular, children with T1D have a markedly increased early mortality associated with hypoglycemia (Gagnum et al., 2015). To avoid severe hypoglycemia, self-monitoring of blood glucose is of utmost importance and finger prick testing or other invasive methods are a prerequisite. The use of continuous glucose monitors (CGMs) is increasing, but they are costly, and worldwide most people with diabetes still rely on pin prick testing. The most dangerous situation for a person with T1D is low blood glucose (BG), and the ability to detect hypoglycemia can be impaired during sleep or other situations with altered consciousness (Secrest et al., 2011; Anderbro et al., 2010). Over time, people with T1D may develop impaired awareness of hypoglycemia (IAH), implying adaptation to repeated hypoglycemic episodes and loss of the ability to sense low BG (Graveling and Frier, 2010). The prevalence of IAH among people with T1D is reported to be $20-25 \%$, and the prevalence increases with time since diagnosis (Geddes et al., 2008; Graveling et al., 2014; Olsen et al 2014). The use of CGMs has demonstrated that hypoglycemic events in people with IAH are more frequent than previously known (Agesen et al., 2018), emphasizing the need for a system that warns them of impending hypoglycemia.

There have been many attempts to develop a non-invasive (NI)BG meter over the last few decades, but barriers still remain (Lin, 2017). Likewise, there has been attempts at developing a hypoglycemia alarm (Schechter et al., 2012; Nguyen et al., 2014; Ling et al., 2016). A simple system based on sweat and temperature sensing has been available for many years, but does not perform at a level that may make it widely used (Clarke et al., 1988). Most approaches so far involve simple sensor systems (i.e. only ECG or skin conductance), without the use of a detection model based on relevant physiology. Moreover, the previous studies have mainly assessed the detection of nocturnal hypoglycemias in people not classified as having IAH, which is less challenging than in those with IAH.

Physiological responses to hypoglycemia are particularly difficult to detect in people with $\mathrm{IAH}$, in whom sympathoadrenal activation occur at a lower BG level than in those with normal hypoglycemia awareness (Hepburn et al., 1991; Elvebakk et al., 2018). Elvebakk 2018 found that global detection (using the same threshold for all cases) of hypoglycemia (down to $2.5 \mathrm{mmol} / \mathrm{L}$ ) in IAH subjects was difficult based on the magnitudes of sudomotor and ECG (heart rate (HR) and QT-interval) responses. The present paper introduces a different approach for detection using a global probabilistic model which evens out individual dependencies and is able to discern episodes of hypoglycemia with better accuracy. In addition, non-invasive prediction of BG is used to further improve the accuracy, presenting the first results on a novel non-invasive multisensory system for detection of hypoglycemia.

The idea for the multisensory system is shown in figure 1. A fall in BG triggers an activation of the sympathetic nervous system, leading to a sudomotor response in innervated dermatomes, increased sympathetic output to the heart and excretion of adrenaline into the bloodstream, consequently increasing the HR and QT-time. Little is known about the sudomotor response to hypoglycemia, in particular which dermatomes of the body that are involved and the differences between individuals, especially between subjects with varying degrees of IAH. Tronstad et al. (Tronstad et al., 2017) showed that amplification of synchronous changes in sudomotor activity (SA) measured at different skin sites (dermatomes) and HR was useful in detecting sympathoadrenal discharge. In IAH sympathoadrenal responses are attenuated (Hepburn et al., 1991) and consequently more difficult to identify (Elvebakk et al., 2018). Thus, new tailored algorithms and adding more information (through sensors) may improve the ability to detect hypoglycemia in this group. One largely independent 
source of information is skin surface sensors for non-invasive prediction of BG (NIBG). Research and development in NIBG has been going on for more than four decades, but the performance still suffers from poor accuracy, physiological time lag and calibration issues (Lin, 2017). Nevertheless, some indication of the BG level (i.e. high or low) and the BG trend (i.e. falling/rising) would provide useful information, and seems possible to obtain using wearable non-invasive sensors (Caduff et al., 2015). Being among the most tried and tested modalities with promising results in this regard is the combination of optical and passive electrical tissue properties measured through the skin. For this application, near-infrared (NIR) and bioimpedance spectroscopy have been selected as candidates for the purpose of increasing the specificity of hypoglycemia detection by reducing the probability for hypoglycemia when the predicted glucose level is too high or lacks a preceding negative trend. In Tronstad et al. (Tronstad et al., 2018), the combination of NIR and bioimpedance was assessed for the non-invasive prediction of BG trends during hypoglycemia. The performance in predicting the BG level was inaccurate with an unreliable threshold-based detection of hypoglycemia, but the trend predictions were mostly able to reflect the fall in glucose toward hypoglycemia. Many factors not related to hypoglycemia may interfere with the sensor measurements, mostly leading to false positive signals by e.g. sympathoadrenal activation through exercise. Some of these confounders are to some extent possible to measure and may provide information which can be used in the algorithm to consider the likelihood of a detected physiological response being spurious. Such sensors include an accelerometer for classification of activity level, one or more skin temperature sensors (for correction of temperature effects in NIBG and contribution in classification of activity levels) and a gyroscope for differentiation between lying and upright positions (different autonomic tone). The accelerometer and skin temperature sensors may also provide information related to hypoglycemia by detection of trembling (Muhlhauser et al., 1991) and skin temperature changes when adrenaline levels are high (Maggs et al., 1994). 


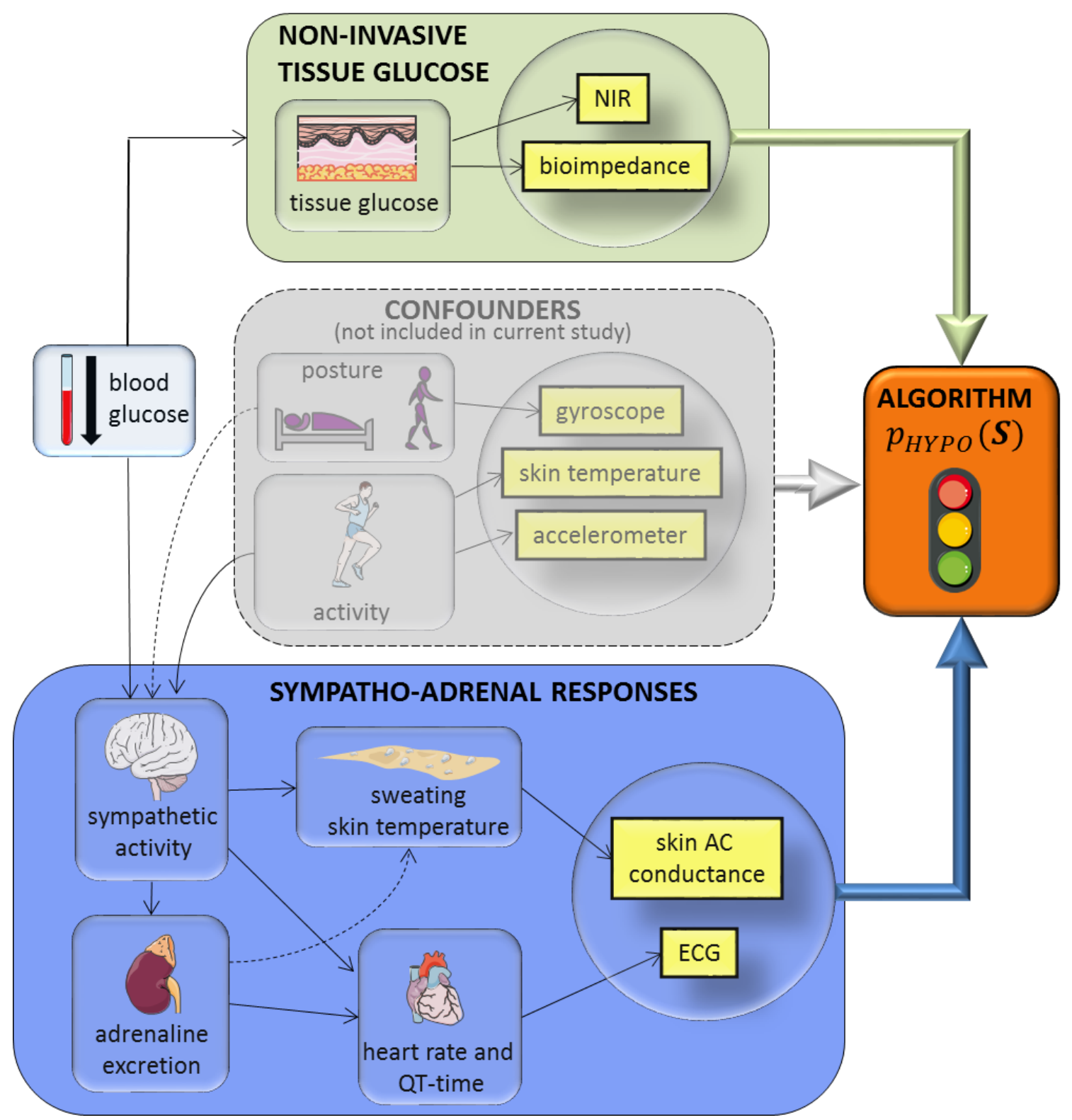

Figure 1. Conceptual illustration of the idea for non-invasive detection of hypoglycemia using a wearable multisensory system. Non-invasive sensors are shown in yellow boxes, each measuring properties which are likely to contribute with relevant information for identifying a hypoglycemic episode. All sensors except the sensors addressing confounders are assessed in the current study, using data from a controlled laboratory environment. (Pictures by Servier medical art (https://smart.servier.com) licensed under CC BY 3.0).

The aim of this study was to develop a model for identification of physiological responses to hypoglycemia in people with T1D and IAH, and to assess the potential for improved non-invasive detection of hypoglycemia in these subjects by including the model in a wearable multisensory system. In this study, we consider all sensor data and knowledge acquired from a glucose clamp study in 20 participants with IAH. Sudomotor activity was recorded at four different skin sites, ECG was measured for HR and QT time, and NIR and bioimpedance spectra were acquired every five minutes, at the same time as blood was drawn for reference glucose measurement. The sensors for identifying confounders (as shown in figure 1) were not used in this study as the subjects were supine in a controlled laboratory environment, but they will be added to future wearable sensor systems and scrutinized in studies focused on detection of spontaneously occurring hypoglycemia. 


\section{Methods}

\section{Participants and glucose clamps}

All sensor and BG data come from the same study of randomized single-blinded euglycemic and hypoglycemic glucose clamps in 20 participants with T1D and IAH, presented in a previous paper (Elvebakk et al., 2018). Briefly, 20 subjects were recruited at Oslo University Hospital, The Norwegian Diabetics Centre and through an article in the Norwegian diabetes journal for patients. Inclusion criteria were age 18-60 years, T1D and IAH (defined by Clarke and/or Gold score (Clarke et al., 1995; Gold et al., 1994)). People with known heart, lung or kidney disease, ECG pathology, other serious medical conditions or the use of medication that could influence any measurements were excluded from the study.

Mean age was $41.1(+/-10.9)$, mean BMI $25.1(+/-2.8)$, mean duration of T1D $23.0(+/-13.8)$ years and mean HbA1c $46.4(+/-8.2) \mathrm{mmol} / \mathrm{mol}$. Twelve of the subjects were women.

Signed informed consent was obtained from all participants, and the study was approved by the regional ethics committee in Norway (REK 2013/813). The sensor equipment setup was evaluated for electrical safety and approved by the appointed committee at Oslo University Hospital.

Each subject participated in two experimental sessions, one hypoglycemic and one euglycemic clamp, separated by at least two weeks. The subjects did not know which type of clamp that would be performed, and the order was randomized so that half of the subjects underwent hypoglycemic clamp first.

Participants were instructed to be extra careful to avoid hypoglycemic episodes 48 hours before each session and were hospitalized the night before clamps to have their BG stabilized with insulin/glucose infusion.

We used a hyperinsulinemic clamp technique with a constant rate insulin infusion of $1.5 \mathrm{mU} / \mathrm{kg} / \mathrm{min}$, and BG was regulated with a variable glucose infusion (Elvebakk et al., 2018).

During hypoglycemic clamp, BG was lowered at a steady pace to $2.5 \mathrm{mmol} / \mathrm{L}$ and stabilized at this level for 15 minutes before being restored to normal levels. During euglycemic clamp, target BG was $5.3 \mathrm{mmol} / \mathrm{L}$. BG was measured every 5 minutes (YSI 2300 STAT Plus glucose analyzer, YSI Life Sciences, Ohio, United States).

\section{Sudomotor activity}

Sudomotor activity was measured by the skin AC conductance method using a Sudologger (BioGauge AS, Oslo, Norway) device having four channels for simultaneous recording at different skin sites. Sensor electrodes of the type 1050NSPM (Covidien) were attached to the measuring sites of the hypothenar eminence of the left hand, the forehead, the abdomen $(2 \mathrm{~cm}$ up and $2 \mathrm{~cm}$ to the right of the umbilicus at the T9 dermatome), and at the palmar wrist of the left arm. Skin AC conductance density (SC) in all channels was recorded four times per second. In order to reflect the activation of the sympathetic nervous system in favor of the interindividual passive electrical properties of the skin, the SC time-series were parameterized into the frequency of skin conductance responses (FSR) as in Tronstad et al. 2017 (Tronstad et al., 2017). Briefly, FSR was calculated by using a five minute moving window, and counting the number of peaks (representing skin conductance responses to sudomotor activity) in the SC time-series within this window, giving a continuous indicator of the average sympathetic nervous system outflow to the different dermatomes where the sensor electrodes were placed. With respect to analysis of detection performance, the FSR time-series were shifted 2.5 minutes back in time so that no yet unknown data points were used in the analysis. Some non-physiologic spike artefacts were present in few recordings but were removed with a custom- 
made spike-removal filter (interpolation between too sharp SC waves). Although the skin AC conductance was measured at the hypothenar eminence of the palm, this sensor was not included in the analysis of hypoglycemia detection, as the electrodermal activity at this site is very influenced by emotional responses and the location is very impractical for a wearable sensor system.

\section{ECG recording for heart rate and QT-interval}

ECG was measured continuously using a Siemens SC9000XL ECG monitor and three Ambu Q00A electrodes in the lead II configuration (which has been suggested as an acceptable substitute for the mean QT-interval from a 12-lead recording (Davey, 2000)). The raw ECG signal was acquired by sampling the analog output of the monitor by a DAQ-card (NI USB 6009 data acquisition device National Instruments, Austin, Tx, USA) connected to a laptop running a custom made LabVIEW (National Instruments, Austin, Tx, USA) application with a sampling rate of $300 \mathrm{~Hz}$.

The raw ECG signals were initially processed by removing any signal parts having a voltage absolute value larger than $1 \mathrm{~V}$ (due to artefacts) and then low pass-filtering using a $3^{\text {rd }}$ order Butterworth filter with a 0.25 Nyquist rate $(37.5 \mathrm{~Hz}$ corner frequency). The $\mathrm{R}$ peaks of the signal were then identified using the Pan-Tompkins algorithm (Pan and Tompkins, 1985), and the time-distance between the neighboring R peaks (the RR-time) was used for the calculation of the HR. Any RR-time lower than $500 \mathrm{~ms}$ or higher than $1500 \mathrm{~ms}$ was excluded due to most likely being an artefact. A heart-rate (HR) time-series was then calculated from the reciprocal of the RR time in seconds and was finally filtered by taking the median within a moving window of 5 minutes.

For calculation of the QT-interval, the bottom of the Q-wave (Q-point) and the end of the T-wave (Tpoint) were located from the ECG signal using a previously published method (Laguna et al., 1990). The QT-interval was then calculated for each heartbeat by the difference between the T-point and the Q-point of the same heartbeat. In order to minimize the influence of the HR variation on the QTinterval, a correction by the Bazett formula (dividing the QT-interval by the square root of the RRinterval in ms) was applied in order to predict the QT-interval at a reference HR of 60 beats per minute (QTC). The Bazett formula was selected in favor of other proposed corrections based on earlier work (Christensen et al., 2010) which found that this formula was most suitable for observing hypoglycemia-induced changes in the QT-interval. The HR-corrected QT-interval time-series were also median filtered using a moving window of 5 minutes. The ECG recording was influenced by some artefacts most likely due to muscle activity, but this influence on HR and QTC was diminished by the median filtering. Similar to the FSR time-series, the HR and QTc time-series were also shifted 2.5 minutes back in time so that no unknown recordings at any time were used in the analysis of hypoglycemia detection.

\section{Skin temperature}

Skin temperature was measured using a Siemens Drager 5204669 temperature probe taped to the upper arm, not covered by clothing. The temperature value was acquired by the ECG monitor (Siemens SC9000XL) connected to the probe and was recorded every 5 minutes at the same time as BG measurement.

\section{NIR and bioimpedance}

NIR and bioimpedance spectroscopy was measured together with the physiological parameters of FSR, HR and QTc for the purpose of increasing the specificity of hypoglycemia. NIR absorbance in the 880-2200 nm range was measured using a Spektron ${ }^{\circledR}$ (Prediktor AS) together with a 6.5 watt tungsten light source (Ocean Optics Inc., Florida, USA) and a custom-made probe attached to the upper arm. The custom-made probe had 6 illumination optical fibers surrounding a central pickup fiber with a $0.2 \mathrm{~mm}$ distance between pickup and illumination fibers. Bioimpedance was measured in 
the $\mathrm{MHz}$ range (1-200 MHz) using a VIA Echo 2500 vector impedance analyzer (AEA Technology, USA) using a 2-electrode configuration with electrodes (Ambu Q00-A) attached at the palmar side of the underarm. During the clamp, NIR and bioimpedance spectra were recorded every five minutes, at the same time as the BG measurement and skin temperature recording. These measurements were used to develop a global model for prediction of BG based on combining NIR, bioimpedance and skin temperature. An artificial neural network regression approach was used to train, validate and test the model and its performance in prediction of BG levels and trends, and is described in its completeness in Tronstad et al. (Tronstad et al., 2019). The predicted BG time-series for each experiment based on the developed model was used in this study for the purpose of improving the specificity of hypoglycemia detection by combining the predicted BG levels and trends with the measured physiological responses.

\section{Probabilistic model for detection of hypoglycemia}

Based on the findings by Elvebakk et al (Elvebakk et al., 2018) and further inspection of the data with respect to temporal properties of the sensors, the model presented in figure 2 was developed. The function of the model is to use a short history of sensor measurements to detect significant withinsubject changes, and to combine the relevant changes from different sensors in a probabilistic network tuned to provide optimal sensitivity and specificity for detecting hypoglycemia.

For each sensor, a moving window of 30 minutes of measurement history was used to determine the probability that a significant change is occurring. The last measurement $m(i)$ in this window is compared to the mean value within the window, calculating the difference and standardized difference as:

$$
\begin{aligned}
& \operatorname{diff}(i)=m(i)-\operatorname{mean}(m(\text { window })) \\
& \text { std.diff }(i)=\frac{m(i)-\operatorname{mean}(m(\text { window }))}{\operatorname{std}(m(\text { window }))}
\end{aligned}
$$

These measures are then converted to a number between 0 and 1 by a sigmoid function where the output $p$ represents the probability of change:

$$
p(x)=\frac{1}{1+e^{k\left(x-x_{0}\right)}}
$$

Where $x$ is the measure (diff or std.diff) and $x_{0}$ is the level of $x$ at which $p(x)$ becomes 0.5 , i.e. a $50 \%$ probability that the change has occurred, and $\mathrm{k}$ is the sharpness or sensitivity to changes around $x_{0}$. In order to ensure that the change is not only statistically sufficient, but also sufficiently large in magnitude of the respective sensor, the probabilities of the difference $\left(p_{\text {diff }}\right)$ and the standardized difference $\left(p_{\text {std.diff }}\right)$ were multiplied (equivalent to a logical AND-gate) for each sensor, expressed as:

$$
p_{\text {sensor }}=p_{\text {diff }} \cdot p_{\text {std.diff }}
$$

The probabilities of reactions in the sudomotor activity sensors ( $p_{\text {forehead }}, p_{\text {abdomen }}$ and $p_{\text {wrist }}$ ) were combined into a total probability of hypoglycemia-related sudomotor activity reaction $\left(p_{F S R}\right)$ by finding the maximum pairwise product:

$$
p_{F S R}(i)=\max \left(p_{\text {Forehead }}(i) \cdot p_{\text {Abdomen }}(i), p_{\text {Forehead }}(i) \cdot p_{\text {Wrist }}(i), p_{\text {Abdomen }}(i) \cdot p_{\text {Wrist }}(i)\right)
$$

For the heart rate, the probability of change was also determined by (1), (2) and (3) and the product of $p_{\text {diff }}$ and $p_{\text {std.diff, }}$, representing the probability of a relevant increase in HR. This probability was then fed through a convolution filter (right half of a Gaussian with a sigma of 7 minutes) in order to smooth the response curves and keep $p_{H R}$ elevated for a few minutes after a significant increase. 
$P_{\text {FSR \&HR }}$ represents the probability of a sympathetic reaction that is due to hypoglycemia based on combining reactions in sudomotor activity $\left(p_{F S R}\right)$ with heart rate in a way that increases the probability if there is a simultaneous heart rate reaction, but goes to zero if there is no sudomotor reaction, regardless of the $p_{H R}$ value (as just standing up will give an increase in HR):

$$
p_{F S R \& H R}=p_{F S R} \cdot\left(1+k \cdot p_{H R}\right)
$$

, where any $p_{\mathrm{FSR} \& H R}>1$ was set to 1 . The probability of QT change associated with hypoglycemia was modeled in the same way using (1) to (4), also smoothed by convolution in the same way as $p_{H R} . P_{Q T}$ was combined with $p_{\text {FSR\&HR }}$ by the following relation, where negative $p_{\text {FSR\&HR\&QT }}$ values were set to zero:

$$
p_{F S R \& H R \& Q T}=p_{F S R \& H R}+P_{Q T}-1
$$

The last step of the model is using the NIBG predictions to filter out responses where the BG is likely to be either too high or lacking a negative foregoing trend. Probabilities of levels ( $\left.p_{\text {level }}\right)$ and trends $\left(p_{\text {trend }}\right)$ in BG associated with hypoglycemia were obtained by sigmoid activation functions as in (3). Due to a low precision of NIBG, the threshold level $\left(\mathrm{x}_{0}\right.$ in (3)) was set to $7.5 \mathrm{mmol} / \mathrm{L}$. The trend was calculated from the slope of a linear regression against time within a 30 minutes window prior to the current measurement, and used to calculate the probability of a relevant trend based on (3) with $x_{0}$ set to $-1 \mathrm{mmol} / \mathrm{L} / \mathrm{h}$. $\mathrm{P}_{\mathrm{NIBG}}$ was calculated based on $p_{\text {level }}$ and $p_{\text {trend }}$ as:

$$
p_{N I B G}=p_{\text {level }}-\left(1-p_{\text {trend }}\right) / 2
$$

This implies that $p_{N I B G}$ is reduced by 0.5 if the trend criterion is not met, and any negative $p_{N I B G}$ is set to zero.

Finally, the probability of physiological responses due to hypoglycemia was modeled by:

\section{$p_{H Y P O}=p_{F S R \& H R \& Q T} \cdot p_{N I B G}$}

In this way, $p_{\text {HYPO }}$ was allowed to remain high unless NIBG indicated too high BG or a lack of a preceding downward glucose trend.

The $k$ value in (3) was set to 1 for all sensors, the $k$ value in (6) was set to 0.5 , and the $x_{0}$ values for calculating $p_{\text {std.diff }}$ for all sensors were also set to 1 (one standard deviation change from the mean gives a $p_{\text {std.diff }}$ of 0.5 ). For $p_{\text {diff, }}$ the $x_{0}$ value was set to 2 for all sudomotor activity sensors and the QT interval, and 3 for the heart rate.

All signal processing and model development were done in Matlab R2018a (Mathworks Inc). 


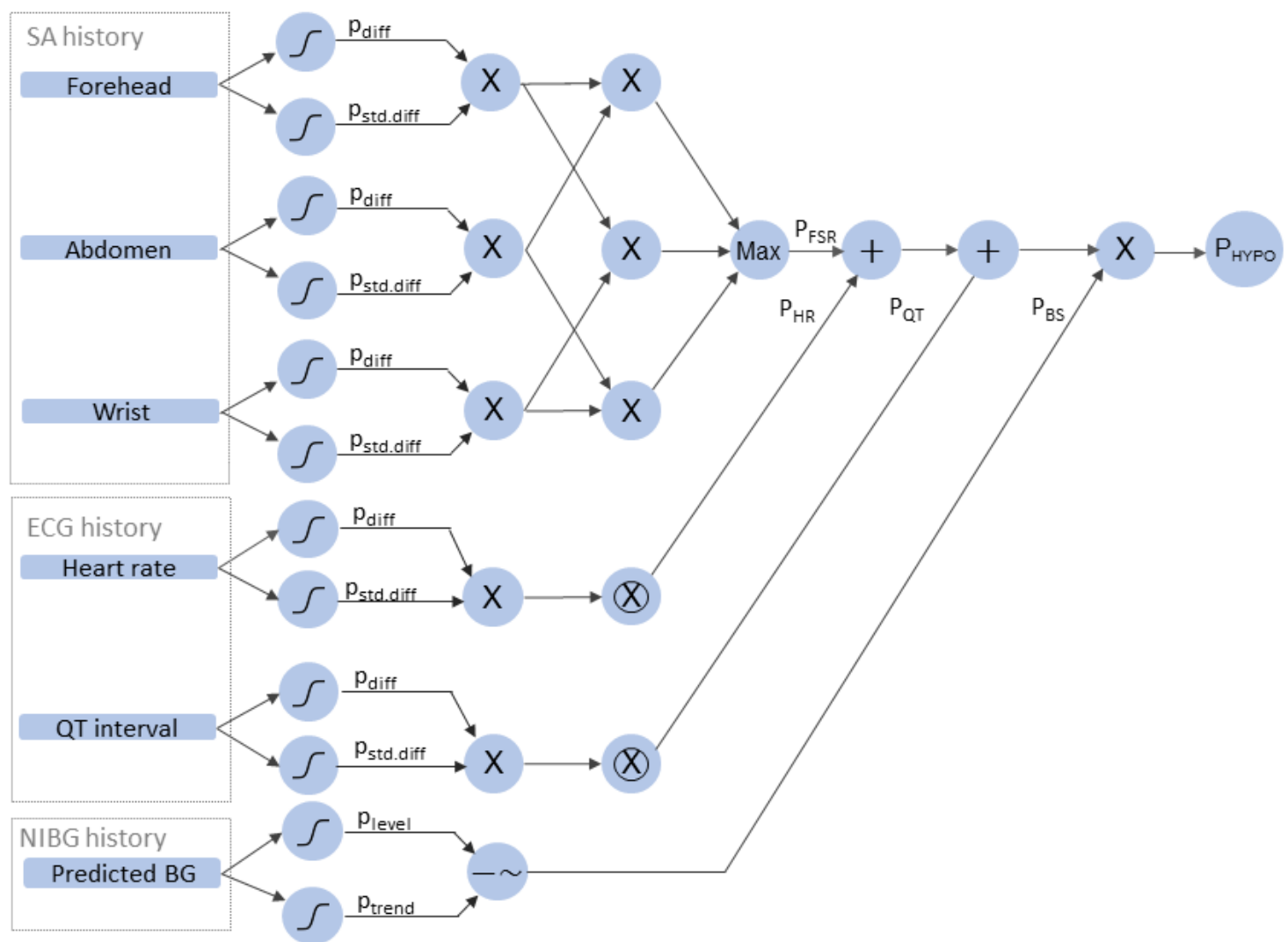

Figure 2. The structure of the probabilistic model used for detection of hypoglycemia based on the input from noninvasive sensors.

\section{Performance assessment}

Based on the probabilistic model and with all sensor recordings from each experiment, $P_{\text {HYPO }}$ was calculated for each time-point from 30 minutes after the beginning of the experiment (due to the history window size) to the end of the recording, corresponding to the BG reference measurements. $P_{\text {HYPO }}$ was then compared to the BG reference measurements, where the intervals having a reference BG below $4.0 \mathrm{mmol} / \mathrm{L}$ were defined as true hypoglycemic intervals. A true positive was counted for at least one $P_{\text {HYPO }}$ above the detection threshold within this interval, else a false negative was counted. Within each of five euglycemic intervals (before and after the hypoglycemic interval of the hypoglycemic clamp, and the three intervals of the euglycemic clamp corresponding in time to the hypoglycemic intervals), a true negative was counted when there was no $P_{\text {HYPO }}$ values above the detection threshold, and a false positive otherwise. Only unique $P_{\text {HYPO }}$ responses were counted in the euglycemic intervals, i.e. not the tail of a response peaking within the hypoglycemic interval.

Sensitivity (same as recall), specificity, precision, F1-score, area under the ROC curve and the median and interquartiles of the detection time (in minutes relative to glucose nadir) was calculated as performance metrics, with the F1-score used as the metric for overall detection accuracy. These metrics were also calculated for different reduced combinations of sensors for comparison, where the same model was used, only with nodes removed from the model structure (see figure 2). When combining only two sudomotor activity sensors, the following formula was used for $p_{F S R}$ :

$$
p_{F S R}=1-\left(1-p_{F S R, \text { Sensor } 1}\right)\left(1-p_{F S R, \text { Sensor } 2}\right)
$$




\section{Results}

The application of the model on sensor data during hypoglycemia is demonstrated in figure 3 . As shown in the b) plot, the sudomotor activity increases around 20 minutes before glucose nadir (the point of lowest BG during the hypoglycemic clamps), both at the forehead and wrist sites. Even though the magnitude increases from nadir and onwards, it is the wave of responses around -10 minutes that is most synchronous (between skin sites) and gives the largest probability value of relevant sudomotor activation, as shown in the d) plot. Shown in e) and f), the probabilities of relevant increases both in HR and QTc are also maxed around this time. Together, these probabilities satisfy the criteria for a non-zero $p_{\text {HYPO }}$ around nadir-10 minutes, which is not attenuated much by the NIBG time-series, having both a low enough predicted glucose level and preceding trend to keep $p_{\text {NIBG }}$ high around this time, as shown in $\mathrm{g})$. The result is a distinct $p_{\text {HYPO }}$ response around nadir -10 minutes as shown in $\mathrm{h}$ ). 
a)

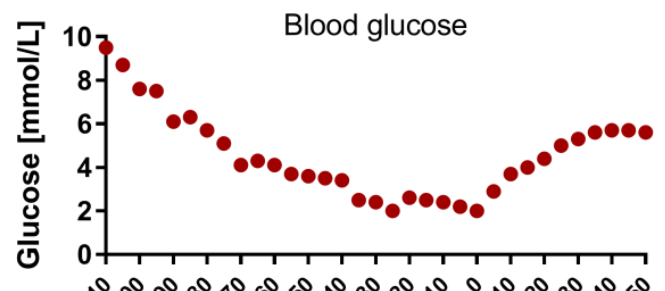

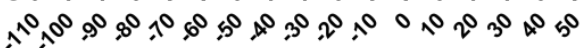

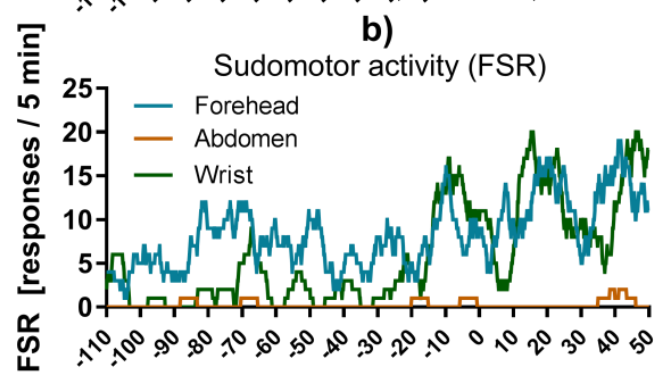

c) - Forehead

$\mathrm{p}_{\mathrm{FSR}} \quad$ - Abdomen

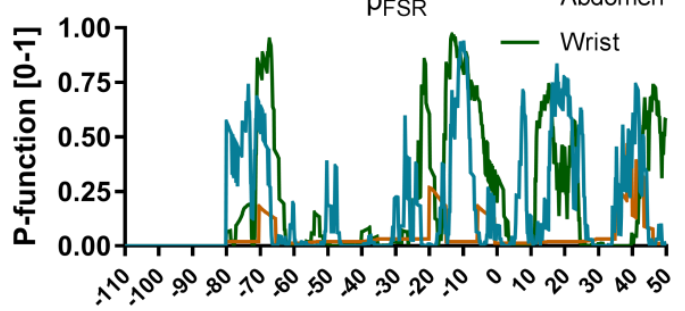

d)

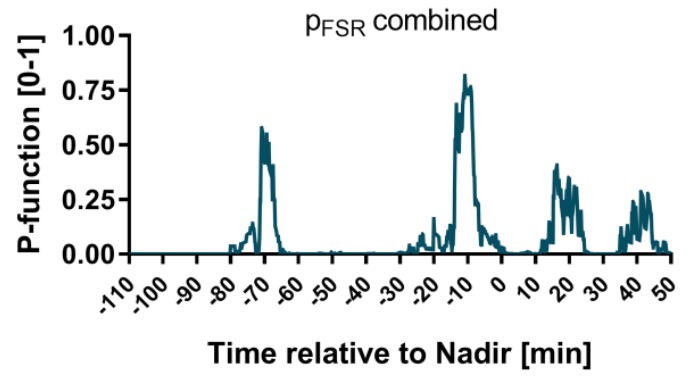

e)

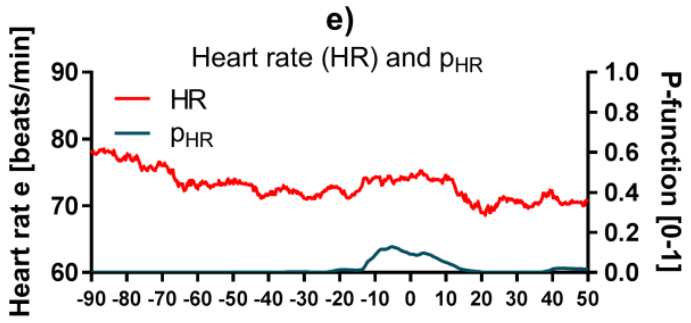

f)

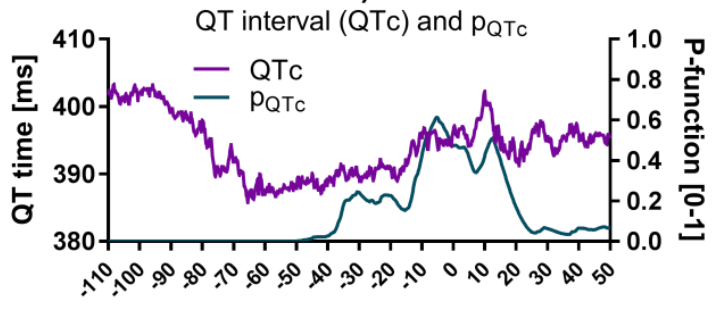

g)

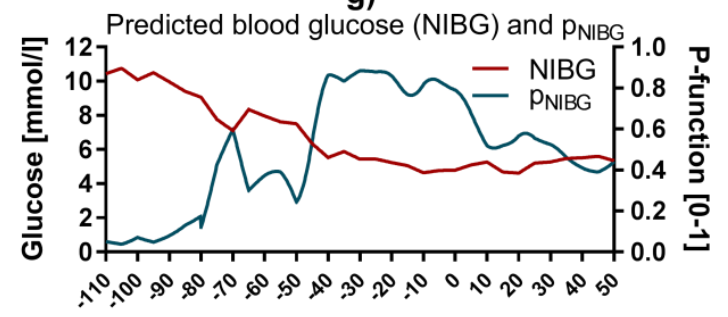

h)

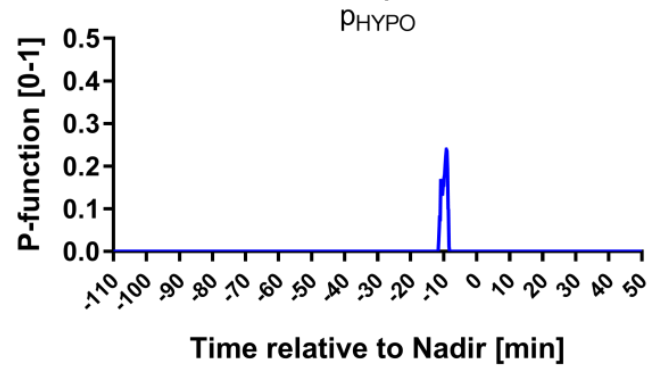

Figure 3. Example of the model applied on sensor data from one hypoglycemic clamp experiment showing the different steps in determining the probability of hypoglycemia $\left(p_{\text {HYPO }}\right)$ using the combination of time-series representing sudomotor activity (b-d), HR (e), QT time (f) and noninvasive prediction of BG $(\mathrm{g})$. Probabilities before -80 minutes before glucose nadir are not available due to the window length required.

The model was able to discern reactions during episodes of hypoglycemia based on the sensor inputs with a high accuracy (F1 score of $88 \%$ ). As shown in figure $4, p_{\text {HYPO }}$ responses were prominent around the time of glucose nadir in the hypoglycemic clamp (upper plot). During the euglycemic clamps, there were two large $p_{\text {HYPO }}$ responses and a few smaller, regarded as false positives depending on the detection threshold level. 


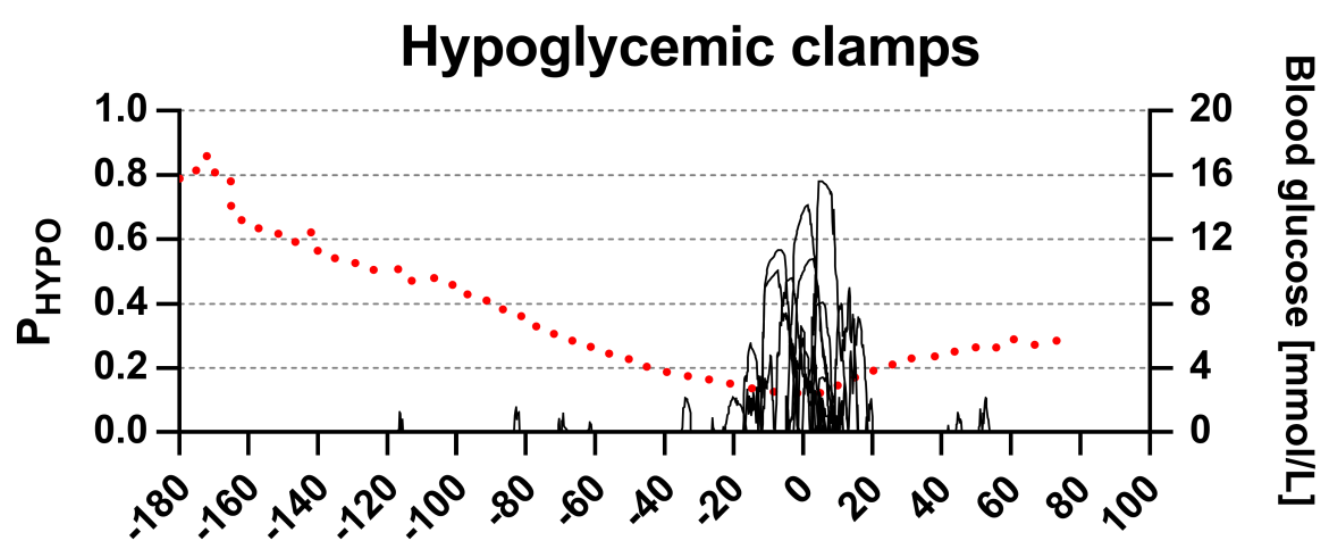

Euglycemic clamps

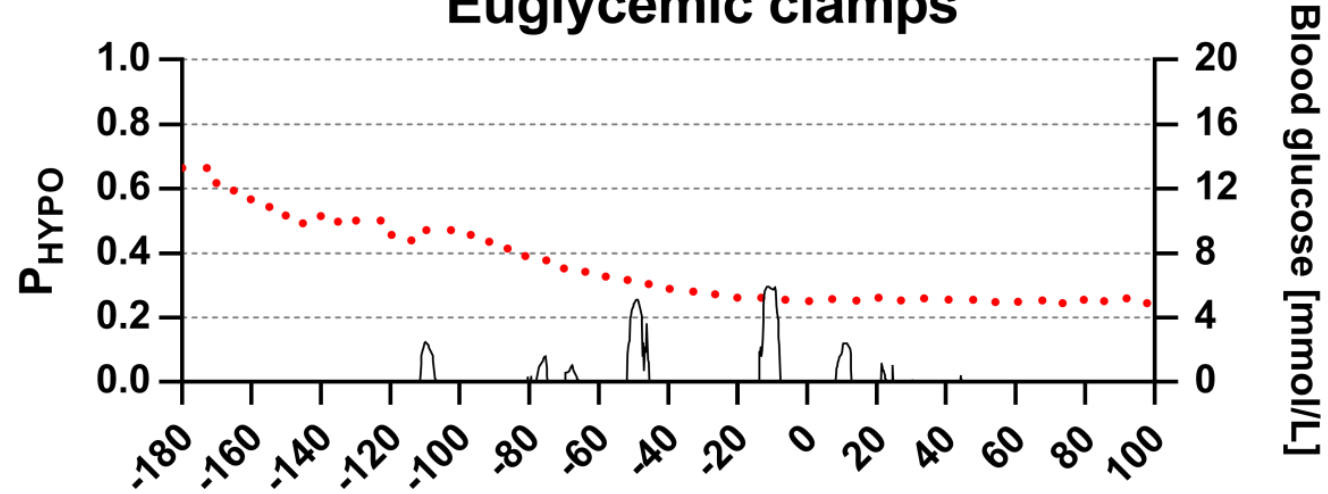

Figure 4. The probability of a physiological response due to hypoglycemia based on the combination of all sensors, $\mathrm{P}_{\mathrm{HYPO}}$, plotted vs time relative to glucose nadir for all hypoglycemic (above) and euglycemic (below) clamps plotted. $P_{\text {HYPO }}$ is represented in black lines together with the mean plasma glucose over all experiments (red dots) on the right y-axis. 


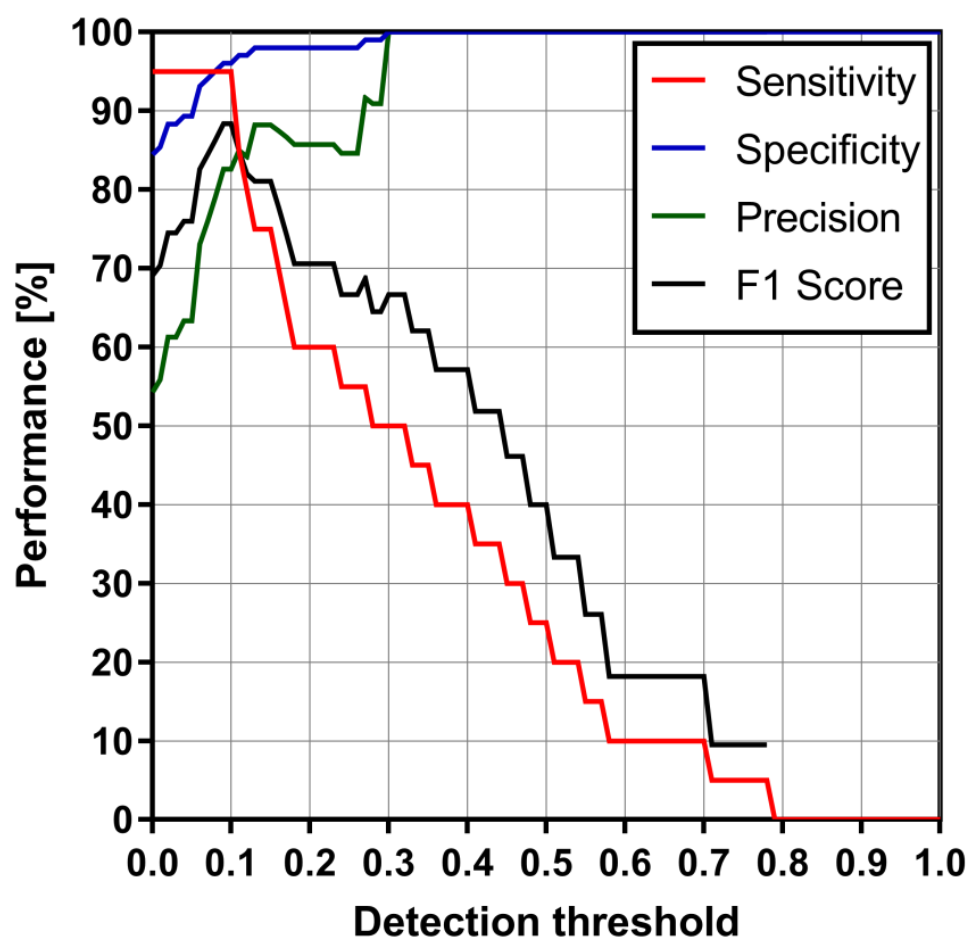

Figure 5. Performance of detection vs threshold of detection. The black line shows the threshold value with the highest $\mathrm{F} 1$-score $(88 \%)$ at $\mathrm{P}_{\mathrm{HYPO}}=0.10$.

The performance of hypoglycemia detection versus detection threshold is shown in figure 5 . The maximum accuracy (based on the F1-score) was with a $P_{\text {HYPO }}$ threshold at 0.10 , with an $88 \%$ accuracy having one false negative and four false positives. Further performance metrics at the optimal detection threshold is shown in table 1, also for other sensor combinations for comparison. Excluding NIBG reduced the accuracy to $86 \%$ (due to lower precision). Interestingly, excluding the HR sensor input did not change the detection performance (except for a bit lower sensitivity and higher precision). A large fall in accuracy was found when the QT time was excluded, hampering both the sensitivity and precision. Using only the abdomen and wrist SA sensors together with both ECG sensors provided an accuracy of $81 \%$. Only using SA data from the wrist site together with both ECG sensors reduced the accuracy to $78 \%$, mainly due to low sensitivity. The combination of only wrist SA together with HR had an accuracy of $55 \%$, with very low precision.

Table 1. Detection performance parameters for the model using all sensors and other selected sensor combinations using the same model. FSR = Frequency of Sudomotor Responses, F=Forehead, $A=A b d o m e n, W=$ Wrist, HR=Heart Rate, $Q T c=H R$-corrected QT-interval, NIBG = Non-Invasive Blood Glucose, IQR = Inter-Quartile Range. The detection time is calculated as the median and interquartiles of the time when an alarm would have triggered in minutes relative to glucose nadir. 


\begin{tabular}{|c|c|c|c|c|c|c|}
\hline Sensors & $\begin{array}{l}\text { Max } \\
\text { F1 } \\
\text { score } \\
{[\%]}\end{array}$ & $\begin{array}{c}\text { Sensitivity } \\
{[\%]}\end{array}$ & $\begin{array}{c}\text { Specificity } \\
{[\%]}\end{array}$ & $\begin{array}{c}\text { Precision } \\
\text { [\%] }\end{array}$ & $\begin{array}{c}\mathrm{AU}_{\mathrm{ROC}} \\
{[0-1]}\end{array}$ & $\begin{array}{c}\text { Detection time } \\
\text { median (IQR) } \\
\text { [min] }\end{array}$ \\
\hline $\mathrm{FSR}(\mathrm{F}+\mathrm{A}+\mathrm{W})+\mathrm{ECG}(\mathrm{HR}+\mathrm{QTC})+\mathrm{NIBG}$ & 88 & 95 & 96 & 83 & 0.97 & $-2.2(-11.6,4.2)$ \\
\hline$F S R(F+A+W)+E C G(H R+Q T c)$ & 86 & 95 & 95 & 79 & 0.96 & $-2.2(-11.6,4.2)$ \\
\hline $\mathrm{FSR}(\mathrm{F}+\mathrm{A}+\mathrm{W})+\mathrm{ECG}(\mathrm{HR})+\mathrm{NIBG}$ & 63 & 65 & 92 & 62 & 0.87 & $-10.8(-27.6,-1.0)$ \\
\hline $\mathrm{FSR}(\mathrm{F}+\mathrm{A}+\mathrm{W})+\mathrm{ECG}(\mathrm{QTC})+\mathrm{NIBG}$ & 86 & 90 & 96 & 82 & 0.97 & $-3.0(-11.8,3.2)$ \\
\hline$F S R(A+W)+E C G(H R+Q T C)+N I B G$ & 83 & 75 & 99 & 94 & 0.94 & $-2.2(-9.2,4.1)$ \\
\hline$F S R(A+W)+E C G(H R+Q T c)$ & 81 & 75 & 98 & 88 & 0.94 & $-2.0(-10.8,6.3)$ \\
\hline$F S R(W)+E C G(H R+Q T c)$ & 78 & 70 & 98 & 88 & 0.91 & $-5.3(-11.8,4.8)$ \\
\hline$F S R(W)+E C G(H R)$ & 55 & 75 & 80 & 43 & 0.80 & $-13.5(-25.9,1.3)$ \\
\hline$E C G(H R+Q T C)+N I B G$ & 58 & 45 & 98 & 82 & 0.79 & $-0.83(-6.2,2.2)$ \\
\hline
\end{tabular}

In Elvebakk et al. (Elvebakk et al., 2018), it was found that five of the 20 subjects could identify hypoglycemia during the clamp experiments. Reducing the sample to the remaining 15 subjects gave a slightly lower performance (F1 score) when using all sensors (87\%), a reduction to $77 \%$ for the two combinations using abdomen and wrist sweating and $74 \%$ for wrist sweating, HR and QTc. A drop in performance was also given for the combination of only FSR wrist and HR combination (51\%), for the combination using all sensors without QTc (59\% F1 score) and for the ECG + NIBG combination (42\%).

\section{Discussion}

\section{Summary of findings}

We have shown that a probabilistic multiparameter model can identify physiological responses during hypoglycemia with a high accuracy using non-invasive sensors, even in people with IAH. Using three SA sensors together with ECG and NIBG, this accuracy was $88 \%$. For a more practical sensor combination with respect to wearability (SA sensors from wrist and abdomen combined with ECG), an accuracy of $81 \%$ was obtained.

\section{Physiological interpretation}

Based on earlier research we were prepared to find that not all subjects would have measurable reactions to a BG of $2.5 \mathrm{mmol} / \mathrm{L}$ (Hepburn et al., 1991). Detecting 19/20 (95\% sensitivity) hypoglycemias in people with IAH would be very attractive if proven to work that well in realistic settings. Since we rely much on a sympathetic reaction, any sympathetic reaction could potentially trigger an alarm. However, none of the false alarms were accompanied by increases in adrenaline or typical symptoms of a sympathetic reaction. The same can be said for some of the alarms during hypoglycemia, since many of the subjects had uncertain reactions to hypoglycemia, yet we could still detect small (possibly sympathetic) sensor reactions in all but one participant. It is therefore difficult to conclude whether our model has detected actual sympathetic reactions during euglycemia (due to other unknown triggers), or random triggering events.

\section{Clinical significance}

One out of 20 hypoglycemic episodes was not detected by our model. Since the sympathetic reaction to hypoglycemia is triggered at a lower BG level in IAH subjects than in people with normal hypoglycemia awareness, this is not surprising. But more importantly, only five out of 20 subjects recognized hypoglycemia during the hypoglycemic clamps. Subtracting one undetected case leaves 14 subjects that had detectable physiological reactions without recognizing these reactions. This may 
imply that even in people with complete hypoglycemia unawareness our device may detect hypoglycemia in time for them to self-treat. However, since one hypoglycemic episode was undetected, it may seem that not only the sympathetic reaction is shifted to a lower BG level in these people, but also the sensors' ability to detect it. This could mean that in some people, it may be impossible to detect hypoglycemia in due time based on these sensors.

Looking at figure 4, we observe that many of the calculated responses to hypoglycemia appear around 10 minutes after glucose nadir. Much, if not all of this can be explained by data processing. A delay of about 10 minutes is not ideal, as BG can fall $0.5 \mathrm{mmol} / \mathrm{L}$ or more during that time period, which might be crucial if the response should appear at around $2.0 \mathrm{mmol} / \mathrm{L}$ and the alarm alerts at $1.5 \mathrm{mmol} / \mathrm{L}$. The model is tuned to achieve optimal sensitivity and precision, but further testing in a real-world setting is needed to decide if other factors should be weighted more.

Today, the CGMs that are available for many people with T1Dare minimally invasive, implying that only a very thin and short plastic catheter penetrates the skin after being inserted via a small needle. A small transmitter device sticks to the skin over the catheter. For many people with T1D such CGM devices are very useful, but IAH continues to be a risk factor for severe hypoglycemia despite the use of CGM (Lin et al., 2019). Also, CGMs imply a considerable cost, and reimbursement by the health care system is variable. For a non-invasive alarm system to be relevant, it would have to be easy to use (CGMs require a minimum of technical interest/knowledge), be non-obtrusive, relatively cheap, and reliant. Modern CGMs are convenient and inobtrusive for most users, while some instantiations of the proposed system would be obtrusive and would benefit from new solutions in sensor design. An advantage of the proposed system is that realizations of the device would not require expensive components, and the use would not require regular replacement of any expensive part, in addition to being non-invasive.

\section{Sensor design}

The presented combinations of sensors may seem obtrusive for a wearable system, especially when using all SA sensors together with ECG and NIBG (NIR and bioimpedance). However, recent and future advances in sensor miniaturization and sensor network architecture will likely reduce the obtrusiveness of a complex multisensory system significantly, combining wireless sensor patches communicating in a body area network. Skin conductance (for SA) can be measured by stamp-size skin patches, as demonstrated by Yoon et al 2016. Miniaturized and wireless ECG patch sensors are already available, such as the SEEQ MCT by Medtronic and the $\mathrm{ZIO}^{\circledR}$ XT Patch by iRhythm. With respect to NIBG sensors, the technological development also facilitates wearable solutions for NIR and bioimpedance (the sensors used in this study). Bioimpedance has the advantage that being an electrical measurement, no other transducer than a suitable electrode is needed, allowing easy sensor miniaturization. ASIC solutions for bioimpedance measurement fabricated in CMOS are emerging (Rodriguez et al., 2016; Zamani et al., 2018; Xu et al., 2015) and wearable patch solutions have been found suitable for bioimpedance sensing (Rossi et al., 2017). Miniature NIR spectrometers in CMOS are also emerging (Hong and Sengupta, 2017), which will be beneficial for application in non-invasive glucose monitoring (Yadav et al., 2015). An integrated circuit for measurement and processing of both NIR and bioimpedance has been proposed (Song et al., 2015), and a wristwatch device with both sensors integrated has already been developed (Carlsen et al., 2016). Advances in research on stretchable materials leads to development of new flexible wearable sensor platforms which may be useful in this application (Kenry et al., 2016). In addition, the developments in the Internet of Things (IoT) will likely pave the way for new solutions in sensor fusion and predictive analytics (Li et al., 2014). 
The model used was global (equally applied to the data from all subjects and experiments in the study), and the detection threshold was the same for all experiments. The detection performance could possibly be improved by personalization with respect to the degree of IAH (i.e. lowering of the detection threshold due to anticipated weaker physiological responses), and a simpler model (with fewer sensors) may provide sufficient detection performance for people with IAH that still have a strong sympathetic reaction (Elvebakk et al., 2018). Studying the effect of personalization would require longer recordings with several hypoglycemic episodes in order to test the reproducibility of individual adjustments.

\section{Comparison to other works}

In our previous study (Elvebakk et al., 2018), we demonstrated that simple processing (as in (Tronstad et al., 2017)) of SA and ECG sensor data and a global threshold could provide detection of hypoglycemia in people with diabetes with normal/adequate hormonal reactions to hypoglycemia (100\% accuracy in five subjects), but had a poor detection performance in people with IAH due to interindividual differences in the magnitude of symptomatic responses. The probabilistic approach presented here leveled out this interindividual variation, mainly by the non-linear transformation in the sigmoid functions and also increasing the sensitivity to weaker responses by the network structure of the model, thereby improving the identification of hypoglycemic responses significantly.

To the authors' knowledge, only one previous study has been published on non-invasive detection of hypoglycemia in people with IAH, and several studies have assessed non-invasive hypoglycemia detection using different sensor systems in people with T1D, but with no consideration of hypoglycemia awareness (see below).

Sejling et al. (Sejling et al., 2015) showed that hypoglycemia could be detected with EEG, and that there was no difference in the EEG signals between subjects with T1D with and without IAH when in the hypoglycemic range. A product called Hypo-Safe is under development, but as of now not commercially available.

Schechter et al (Schechter et al., 2012) used a sensor system of HR, perspiration (unprocessed skin conductance at the fingertips), skin temperature and tremor (by accelerometer) measurements and obtained a sensitivity of $100 \%$ and a specificity of $85.7 \%$ in detecting nocturnal hypoglycemia in a pilot study in ten adolescents with type 1 diabetes. Clewett et al. demonstrated feasibility of noninvasive EEG-based hypoglycemia warning in a single case study (Clewett et al., 2016).

Recently, machine-learning approaches for hypoglycemia detection have also been attempted (Nguyen et al., 2014, Huang et al., 2011, Ling et al., 2016, Marling et al., 2016) based on similar sensors such as ECG, skin conductance and temperature, but are difficult to compare with the present study due to differences in population, study design and performance measures. It seems that too little data from too few studies have so far been gathered in order to evaluate the potential for non-invasive detection of hypoglycemia using machine-learning methods.

Previous studies involving skin conductance (or other names thereof, such as galvanic skin reflex or electrodermal activity) have used the raw measurement without any parameterization. In the present study, signal processing of this measurement was employed in order to extract the FSR (frequency of sudomotor responses), reducing the between-subjects variation due to skin properties and more closely representing the state of sympathoadrenal activation (Tronstad et al., 2017).

\section{Limitations of the present study}

Although the dataset used in this study is large compared to other studies ( 40 glucose clamp experiments in 20 subjects), data scarcity is also considered a limit of this study. Especially in the IAH 
population, large interindividual differences in hormonal reactions to hypoglycemia and the associated physiological responses necessitate a large sample. Although machine-learning was not employed in the present study, some manual tuning of several parameters (i.e. $x_{0}$ in (3)) was done in order to translate relevant changes in sensor measurements into reasonable probabilities for their respective physiological parameter, implying some risk of overfitting. In addition, the structure of the model (figure 2) was developed by a combination of logical reasoning and optimization of global detection accuracy on this dataset. Hence, this is an introduction of a model for identification of physiological responses due to hypoglycemia, an assessment of its potential for detecting hypoglycemia, but not a validation of it.

A strength of this study, however, is the participant-blinded randomized control clamp that provided measurements during similar conditions but at euglycemic levels, greatly reducing the chance of overfitting and spurious relationships. It is also not possible to know whether all detected responses within the hypoglycemic window are causally related to BG decrease, especially those who peak more than 10 minutes after glucose nadir (see figure 4). Given the latency due to signal processing, a possible latency in the physiological response to hypoglycemia and the lack of time-corresponding responses in the euglycemic clamp, we believe that they are most likely a consequence of hypoglycemia. Ideally, the model would be tuned further on a larger sample from the same population with data from several hypoglycemic episodes per subject, and then validated on a new sample from the same population. The measurements in this study were done during glucose clamp in a controlled laboratory environment in order to study the physiological responses to hypoglycemia as specifically as possible. Using a wearable device with the same sensors as described in figure 1 , the detection performance can be evaluated in a more realistic setting during daily activities and during sleep. Further studies should also include an assessment of the potential for reductions in uncorrected hypoglycemic episodes and a comparison with recent CGM technology.

\section{Conclusion}

In conclusion, we have presented a novel model for identification of non-invasively measurable physiological responses related to hypoglycemia, showing potential for the detection of moderate hypoglycemia in people with T1D and IAH using a wearable sensor system.

\section{Acknowledgements}

This work was supported by funding from the South-Eastern Norway Regional Health Authority and the Norwegian Diabetes Association.

The Authors would like to thank all study participants and nurses involved in the study, in particular Åse Halsne, Gørild Vinje, Karin Eide and Gro Boezelijn, nurses at the Diabetes Laboratory, Oslo University Hospital, for assistance during the clamping experiments.

\section{References}

Agesen R M, Kristensen P L, Beck-Nielsen $H$, Norgaard K, Perrild H, Jensen T, Parving H H, Thorsteinsson B, Tarnow L and Pedersen-Bjergaard U 2018 Effect of Insulin Analogs on Frequency of Non-Severe Hypoglycemia in Patients with Type 1 Diabetes Prone to Severe Hypoglycemia: Much Higher Rates Detected by Continuous Glucose Monitoring than by SelfMonitoring of Blood Glucose-The HypoAna Trial Diabetes technology \& therapeutics $20247-$ 56 
Anderbro T, Amsberg S, Adamson U, Bolinder J, Lins P E, Wredling R, Moberg E, Lisspers J and Johansson U B 2010 Fear of hypoglycaemia in adults with Type 1 diabetes Diabet Med 27 1151-8

Caduff A, Zanon M, Mueller M, Zakharov P, Feldman Y, De Feo O, Donath M, Stahel W A and Talary M S 2015 The Effect of a Global, Subject, and Device-Specific Model on a Noninvasive Glucose Monitoring Multisensor System J Diabetes Sci Technol 9 865-72

Carlsen S, Fougner A, Vegar Karstang T, Staal O and Christian Christiansen S 2016 Continuous NonInvasive Glucose Monitoring by Sensor Fusion of Near Infrared Light and Bioimpedance Measurements: Results of a Proof of Concept Study Conference on Advanced Technologies \& Treatments for Diabetes (ATTD) Milano, Italy. DOI: 10.13140/RG.2.2.34384.10247

Christensen T F, Randlov J, Kristensen L E, Eldrup E, Hejlesen O K and Struijk J J 2010 QT Measurement and Heart Rate Correction during Hypoglycemia: Is There a Bias? Cardiol Res Pract 2010961290

Clarke W L, Carter W R, Moll M, Cox D J, Gonder-Frederick L A and Cryer P E 1988 Metabolic and cutaneous events associated with hypoglycemia detected by sleep sentry Diabetes Care $\mathbf{1 1}$ 630-5

Clarke W L, Cox D J, Gonder-Frederick L A, Julian D, Schlundt D and Polonsky W 1995 Reduced awareness of hypoglycemia in adults with IDDM. A prospective study of hypoglycemic frequency and associated symptoms Diabetes Care 18 517-22

Clewett C J, Langley P, Bateson A D, Asghar A and Wilkinson A J 2016 Non-invasive, home-based electroencephalography hypoglycaemia warning system for personal monitoring using skin surface electrodes: a single-case feasibility study. In: Healthcare Technology Letters: Institution of Engineering and Technology) pp 2-5

Davey P P 2000 Which lead for Q-T interval measurements? Cardiology 94 159-64

Elvebakk O, Tronstad C, Birkeland K I, Jenssen T G, Bjorgaas M R, Froslie K F, Godang K, Kalvoy H, Martinsen O G and Gulseth H L 2018 Evaluation of Hypoglycaemia with Non-Invasive Sensors in People with Type 1 Diabetes and Impaired Awareness of Hypoglycaemia Sci Rep 814722

Gagnum V, Stene L C, Sandvik L, Fagerland M W, Njolstad P R, Joner G and Skrivarhaug T 2015 Allcause mortality in a nationwide cohort of childhood-onset diabetes in Norway 1973-2013 Diabetologia 58 1779-86

Geddes J, Schopman J E, Zammitt N N and Frier B M 2008 Prevalence of impaired awareness of hypoglycaemia in adults with Type 1 diabetes Diabet Med 25 501-4

Gold A E, MacLeod K M and Frier B M 1994 Frequency of severe hypoglycemia in patients with type I diabetes with impaired awareness of hypoglycemia Diabetes Care 17 697-703

Graveling A J and Frier B M 2010 Impaired awareness of hypoglycaemia: a review Diabetes \& metabolism 36 Suppl 3 S64-74

Graveling A J, Noyes K J, Allerhand M H, Wright R J, Bath L E, Deary I J and Frier B M 2014 Prevalence of impaired awareness of hypoglycemia and identification of predictive symptoms in children and adolescents with type 1 diabetes Pediatr Diabetes 15 206-13

Hepburn D A, Patrick A W, Brash H M, Thomson I and Frier B M 1991 Hypoglycaemia unawareness in type 1 diabetes: a lower plasma glucose is required to stimulate sympatho-adrenal activation Diabet Med 8 934-45

Hong L and Sengupta K 2017 Fully Integrated Optical Spectrometer in Visible and Near-IR in CMOS IEEE Trans Biomed Circuits Syst 11 1176-91

Huang G-B, Hui Wang D and Lan Y 2011 Extreme learning machines: a survey. Int J Mach Learn Cybern vol 2

Kenry, Yeo J C and Lim C T 2016 Emerging flexible and wearable physical sensing platforms for healthcare and biomedical applications Microsystems \&Amp; Nanoengineering 216043

Laguna P, Thakor N V, Caminal P, Jane R, Yoon H R, Bayes de Luna A, Marti V and Guindo J 1990 New algorithm for QT interval analysis in 24-hour Holter ECG: performance and applications Med Biol Eng Comput 28 67-73

Li S, Xu L D and Zhao S 2014 The internet of things: a survey Information Systems Frontiers 1716 
Lin T 2017 Non-Invasive Glucose Monitoring: A Review of Challenges and Recent Advances vol 6

Lin Y K, Hung M, Sharma A, Chan O, Varner M W, Staskus G and Fisher S J 2019 Impaired awareness of hypoglycemia continues to be a risk factor for severe hypoglycemia despite the use of continuous glucose monitoring system in type 1 diabetes Endocrine Practice 25 517-525.

Lind M, Svensson A M, Kosiborod M, Gudbjornsdottir S, Pivodic A, Wedel H, Dahlqvist S, Clements M and Rosengren A 2014 Glycemic control and excess mortality in type 1 diabetes N Engl J Med 371 1972-82

Ling S H, San P P and Nguyen H T 2016 Non-invasive hypoglycemia monitoring system using extreme learning machine for Type 1 diabetes ISA transactions 64 440-6

Livingstone S J, Looker H C, Hothersall E J, Wild S H, Lindsay R S, Chalmers J, Cleland S, Leese G P, McKnight J, Morris A D, Pearson D W, Peden N R, Petrie J R, Philip S, Sattar N, Sullivan F and Colhoun H M 2012 Risk of cardiovascular disease and total mortality in adults with type 1 diabetes: Scottish registry linkage study PLoS medicine 9 e1001321

Maggs D G, Macdonald I A and Tattersall R B 1994 Thermoregulatory responses to hyperinsulinaemic hypoglycaemia and euglycaemia in IDDM Diabetologia 37 689-96

Marling C, Xia L, Bunescu R and Schwartz F L 2016 Machine Learning Experiments with Noninvasive Sensors for Hypoglycemia Detection. In: Proceedings of IJCAI 2016 Workshop on Knowledge Discovery in Healthcare Data, pp 1-6

Muhlhauser I, Heinemann L, Fritsche E, von Lennep K and Berger M 1991 Hypoglycemic symptoms and frequency of severe hypoglycemia in patients treated with human and animal insulin preparations Diabetes Care 14 745-9

Nguyen L L, Su S and Nguyen H T 2014 Neural network approach for non-invasive detection of hyperglycemia using electrocardiographic signals Conference proceedings : ... Annual International Conference of the IEEE Engineering in Medicine and Biology Society. IEEE Engineering in Medicine and Biology Society. Annual Conference 2014 4475-8

Olsen SE, Asvold BO, Frier BM, Aune SE, Hansen LI, Bjørgaas MR. 2014 Hypoglycaemia

symptoms and impaired awareness of hypoglycaemia in adults with Type 1 diabetes: the association with diabetes duration. Diabet Med 31 1210-7.

Pan J and Tompkins W J 1985 A real-time QRS detection algorithm IEEE Trans Biomed Eng 32 230-6

Rodriguez S, Ollmar S, Waqar M and Rusu A 2016 A Batteryless Sensor ASIC for Implantable BioImpedance Applications IEEE Transactions on Biomedical Circuits and Systems 10 533-44

Rossi S, Mancarella C, Mocenni C and Torre L D 2017 Bioimpedance sensing in wearable systems: From hardware integration to model development. In: 2017 IEEE 3rd International Forum on Research and Technologies for Society and Industry (RTSI), pp 1-6

Schechter A, Eyal O, Zuckerman-Levin N, Amihai-Ben-Yaacov V, Weintrob N and Shehadeh N 2012 A prototype of a new noninvasive device to detect nocturnal hypoglycemia in adolescents with type 1 diabetes--a pilot study Diabetes technology \& therapeutics 14 683-9

Secrest A M, Becker D J, Kelsey S F, LaPorte R E and Orchard T J 2010 All-cause mortality trends in a large population-based cohort with long-standing childhood-onset type 1 diabetes: the Allegheny County type 1 diabetes registry Diabetes Care 33 2573-9

Secrest A M, Becker D J, Kelsey S F, Laporte R E and Orchard T J 2011 Characterizing sudden death and dead-in-bed syndrome in Type 1 diabetes: analysis from two childhood-onset Type 1 diabetes registries Diabet Med 28 293-300

Sejling A S, Kjaer T W, Pedersen-Bjergaard U, Diemar S S, Frandsen C S, Hilsted L, Faber J, Holst J J, Tarnow L, Nielsen M N, Remvig L S, Thorsteinsson B and Juhl C B 2015 Hypoglycemiaassociated changes in the electroencephalogram in patients with type 1 diabetes and normal hypoglycemia awareness or unawareness Diabetes 64 1760-9

Song K, Ha U, Park S, Bae J and Yoo H 2015 An Impedance and Multi-Wavelength Near-Infrared Spectroscopy IC for Non-Invasive Blood Glucose Estimation IEEE Journal of Solid-State Circuits 50 1025-37

Tronstad C, Elvebakk O, Kalvoy H, Bjorgaas M R and Martinsen O G 2017 Detection of sympathoadrenal discharge by parameterisation of skin conductance and ECG measurement 
Conference proceedings : ... Annual International Conference of the IEEE Engineering in Medicine and Biology Society. IEEE Engineering in Medicine and Biology Society. Annual Conference 2017 3997-4000

Tronstad C, Elvebakk O, Staal O M, Kalvøy H, Høgetveit J O, Jenssen T G, Birkeland K I and Martinsen $\varnothing \mathrm{G} 2019$ Non-invasive prediction of blood glucose trends during hypoglycemia Analytica Chimica Acta 1052 37-48

Xu J, Harpe P, Pettine J, Hoof C V and Yazicioglu R F 2015 A low power configurable bio-impedance spectroscopy (BIS) ASIC with simultaneous ECG and respiration recording functionality. In: ESSCIRC Conference 2015 - 41st European Solid-State Circuits Conference (ESSCIRC), pp 396-9

Yadav J, Rani A, Singh V and Murari B M 2015 Prospects and limitations of non-invasive blood glucose monitoring using near-infrared spectroscopy Biomedical Signal Processing and Control 18 214-27

Zamani M, Rezaeiyan Y, Shoaei O and Serdijn W A 2018 A $1.55 \mu$ W Bio-Impedance Measurement System for Implantable Cardiac Pacemakers in $0.18 \mu \mathrm{m}$ CMOS IEEE Transactions on Biomedical Circuits and Systems 12 211-21 